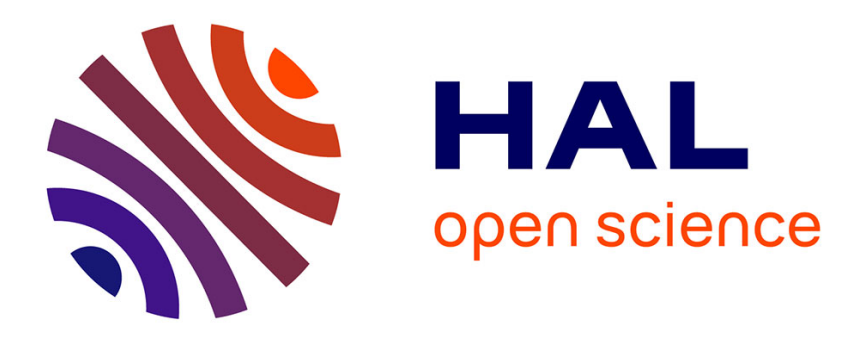

\title{
On Peres approach to Fradkin-Bacry-Ruegg-Souriau's perihelion vector
}

Yves Grandati, Alain Berard, Herve Mohrbach

\section{To cite this version:}

Yves Grandati, Alain Berard, Herve Mohrbach. On Peres approach to Fradkin-Bacry-Ruegg-Souriau's perihelion vector. 2008. hal-00331850

\section{HAL Id: hal-00331850 \\ https://hal.science/hal-00331850}

Preprint submitted on 21 Oct 2008

HAL is a multi-disciplinary open access archive for the deposit and dissemination of scientific research documents, whether they are published or not. The documents may come from teaching and research institutions in France or abroad, or from public or private research centers.
L'archive ouverte pluridisciplinaire HAL, est destinée au dépôt et à la diffusion de documents scientifiques de niveau recherche, publiés ou non, émanant des établissements d'enseignement et de recherche français ou étrangers, des laboratoires publics ou privés. 


\title{
On Peres approach to Fradkin-Bacry-Ruegg-Souriau's perihelion vector
}

\author{
Y. Grandati, A. Bérard and and H. Mohrbach \\ Laboratoire de Physique Moléculaire et des Collisions, \\ ICPMB, IF CNRS 2843, Université Paul Verlaine, \\ Institut de Physique, Bd Arago, 57078 Metz, Cedex 3, France
}

\begin{abstract}
We solve explicitely the differential system obtained by Peres for the construction of a conserved vector associated to any central potential. We then obtain a very direct access to the discontinuous behavior of this Fradkin-Bacry-Ruegg-Souriau perihelion vector.
\end{abstract}

PACS numbers:

\section{INTRODUCTION}

Since the pioneer works of Ermanno, Bernouilli and Laplace during eighteenth century国四 we know that the Kepler system presents a specific vector conserved quantity, the so-called Laplace-Runge-Lenz vector, which provides in particular a very simple access to the orbit equation. Today, the existenfe of generalized Laplace-Runge-Lenz vectors for various extensions of the Kepler problem have been put in evidencel.

This supplementary constant of motion has a dynamical origif systems, of a symmetry group larger than the space-time's one case and by Fradkin 10 in the 3D case, the isotropic harmonic oscillator shares the same feature and admits also a supplementary dynamicalconserved quantity but of tensorial type, the Fradkin-Jauch-Hill tenson] 6.1 . 1 .

Bacry, Ruegg, Souriau 12 and Fradkin 13 made an important additional step when they showed that all three dimensional dynamical problems involving central potentials possess the extended symmetry algebra $\mathrm{O}_{4}$ and $\mathrm{SU}_{3}$, a result soon generalized by Mukunda 14 for any hamiltonian system with n degrees of freedom. As noted by Bacry, Ruegg and Souriau in their seminal paper 22, the general ability to construct such a local Lie algebra of dynamical symmetry is not surprising since all the $2 \mathrm{n}$-dimensional symplectic manifolds are locally isomorphic 5 . However the following step to ensure a global dynamical symmetry is to verify that the finite canonical transformations generated by this algebra form a group, a feature of the Kepler problem. As established by Stehle and Han 16 , the existence of such a "global" higher symmetry group, admitting finitely multivalued realizations on the phase space of the system, is a necessary condition for the existence of degeneracies.

Bacry, Ruegg, Souriau12, as much as Fradkin 13 , gave an explicit construction scheme for a vector with constancy properties associated to every central potential, Fradkin's derivation being very direct and explicit. Bacry, Ruegg and Souriau already noted that such a vector is exceptionally one-valued, as in the Kepler case. The first explicit demonstration of the multivalud behavior of these Fradkin-Bacry-Ruegg-Souriau (FBRS) vectors have been established by Serebrennikov, Shabad 17 , Buch and Denman 18 when these authors pointed out that in specific cases Fradkin vectors are in fact only piecewise conserved.

In 1979, Peres 19 , using a distinct approach, rediscovered the FBRS generalized Laplace-Runge-Lenz vector. He obtained a differential system for the coefficients of the vectors which presents singularities at the apsidal positions. Without solving explicitely the differential system, Peres infered from this singular behavior the existence of discontinuous changes of direction of the vector which is then_nnly piecewise constant. The correspondence between Fradkin's and Peres approaches have been studied by Yoshida20.

A complete answer to the question concerning the status of conserved quantity which could be attributed to the FBRS vector has been given by Holas and March 21. They showed explicitely that at each pericenter (or apocenter, depending on the chosen initial conditions), the FBRS vector changes its direction abruptly from an angle double of the apsidal one. A lastattempt to obtain a "true" vector constant of motion in every conservative central force field has been made by Yan22 but, as shown a short time after by Holas and March23, Yan's construction coincides in fact with the FBRS perihelion vector and then presents the same discontinuities. The generalized Laplace-Runge-Lenz vector can also be deduce in a direct and elegant manner from the equation of motiont

Note finally that, in a very recent contribution, Ballesteros and al.24 use FBRS type vectors to establish an optimal extension of Bertrand's theorem 25,26 to curved spaces.

In this paper, we give an explicit solution to the differential system obtained by Peres 19 . Using then a complex formulation, particularly convenient for the treatment of planar problems 27.28, we recover in a very simple manner the discontinuous behavior of the FBRS vector studied by Holas and Marche1. 


\section{BASICS OF 2D MOTION IN A CENTRAL POTENTIAL}

\section{Complex formulation}

We consider a planar motion $\vec{r}(t)=\left(\begin{array}{l}x(t) \\ y(t)\end{array}\right)_{\left(O, \overrightarrow{u_{x}}, \overrightarrow{u_{y}}\right)}$ for a particle of mass $m=1$ submitted to a potential $U(\vec{r})$, eventually singular at the origin. We choose to adopt a complex formulation where we represent the position by its corresponding affix $z(t)=x(t)+i y(t)$, the potential being then viewed as a real valued function of $z, U(z, \bar{z})$, defined on $\mathbb{C}$ or $\mathbb{C}^{*}$. The gradient of any real valued function of $z$ and $\bar{z}$ is then given by :

$$
\vec{\nabla} U(\vec{r}) \rightarrow 2 \frac{\partial U(z, \bar{z})}{\partial \bar{z}}
$$

where $\frac{\partial}{\partial \bar{z}}=\overline{\frac{\partial}{\partial z}}=\frac{1}{2}\left(\frac{\partial}{\partial x}+i \frac{\partial}{\partial y}\right)$.

The equation of motion for our system takes the form:

$$
\ddot{z}+2 \frac{\partial U(z, \bar{z})}{\partial \bar{z}}=0
$$

where the dot represents the time derivative.

For two vectors $\vec{A}$ and $\vec{B}$ in the $\left(O, \overrightarrow{u_{x}}, \overrightarrow{u_{y}}\right)$ plane, $\vec{A} \times \vec{B}$ is represented in complex form by the real quantity $\operatorname{Im}(\bar{A} B), A$ and $B$ be being the complex affixes of $\vec{A}$ and $\vec{B}$ respectively.

The angular momentum (which necesseraly conserves its direction orthogonal to the plane) $\vec{L}(t)=\vec{r}(t) \times \dot{\vec{r}}(t)=$ $L(t) \vec{k}$, admits then the following correspondent :

$$
L=\operatorname{Im}(\bar{z}(t) \dot{z}(t))=\frac{1}{2 i}(\bar{z} \dot{z}-\dot{\bar{z}} z)
$$

In the case of a central potential $U(z, \bar{z})=U(|z|)=U(r)$, we have:

$$
\vec{\nabla} U(r) \rightarrow \frac{z}{r} U^{\prime}(r)
$$

where $f^{\prime}(x)=\frac{d f(x)}{d x}$ is the usual derivative of a function $f$ of one variable $x$.

Eq.(2) becomes simply:

$$
\ddot{z}+\frac{z}{r} U^{\prime}(r)=0
$$

Using this result, the angular momentum's conservation is immediate (see Eq.(3)):

$$
\dot{L}=\frac{1}{2 i}(\bar{z} \ddot{z}-\ddot{\bar{z}} z)=0
$$

\section{Radial equation of motion}

If we use a polar representation for $z, z=r e^{i \theta}$, with $\theta \in[0,2 \pi[$, we have:

$$
\widehat{\left(r^{2}\right)}=2 \dot{r} r=\dot{z} \bar{z}+z \dot{\bar{z}}
$$

which gives:

$$
\dot{r}=\frac{1}{2 r}(\dot{z} \bar{z}+z \dot{\bar{z}})=\frac{1}{r}(z \dot{\bar{z}}+i L)
$$


and $\left(\right.$ since $\left.e^{2 i \theta}=\frac{z}{\bar{z}}\right)$ :

$$
\dot{\theta}(t)=\frac{\bar{z} \dot{z}-\dot{\bar{z}} z}{2 i r^{2}}=\frac{L}{r^{2}(t)}
$$

Then, we can write:

$$
\dot{z}=\dot{r} e^{i \theta}+i \dot{\theta} r e^{i \theta}=\left(\dot{r}+i \frac{L}{r}\right) \frac{z}{r}
$$

and:

$$
\ddot{z}=\left(\frac{\ddot{r}}{r}-\frac{L^{2}}{r^{4}}\right) z
$$

Then using Eq.(5), we deduce an equation for the the radial motion:

$$
\ddot{r}(t)=\frac{L^{2}}{r^{3}(t)}-U^{\prime}(r(t))
$$

which is readily integrated as :

$$
(\dot{r})^{2}=2\left(E-\frac{L^{2}}{2 r^{2}}-U(r)\right)
$$

$E$ is a constant of integration, which can be identified with the energy of the system, since from Eq.(10) we have:

$$
\frac{1}{2}|\dot{z}|^{2}=\frac{1}{2}\left(\frac{\dot{r}}{r}+i \frac{L}{r^{2}}\right) z\left(\frac{\dot{r}}{r}-i \frac{L}{r^{2}}\right) \bar{z}=\frac{1}{2}\left((\dot{r})^{2}+\frac{L}{r^{2}}\right)=E-U(r)
$$

If we note $T(r)=E-U(r)$, Eq.(12) takes the form:

$$
(\dot{r}(t))^{2}=\frac{L^{2}}{r^{2}} f(r(t))
$$

where :

$$
f(r)=\frac{2 r^{2}}{L^{2}} T(r)-1=\frac{2 r^{2}}{L^{2}}\left(E-V_{L}(r)\right),
$$

$V_{L}(r)=U(r)+\frac{L^{2}}{2 r^{2}}$ being the radial effective potential.

From Eq. (15) and Eq.(16), we see that the radial oscillation motion presents, for bounded orbits, two turning point $r_{m}$ and $r_{M}$, corresponding to the pericenters et apocenters (apsidal positions) of the orbital motion. They are roots of the equation:

$$
f(r)=0 \Leftrightarrow V_{L}(r)=E
$$

In the sequel, we will suppose that $r_{m}$ and $r_{M}$, are simple roots, that is $V_{L}^{\prime}\left(r_{m}\right)<0$ and $V_{L}^{\prime}\left(r_{M}\right)<0$.

At the apsidal positions, $\dot{r}_{m}=r_{M}=0$ and:

$$
\dot{z}_{j}=i \dot{\theta}_{j} z_{j}=i \frac{L}{r_{j}^{2}} z_{j}, \quad j=m, M
$$


The $i$ factor in the right hand side of Eq.(18) implies that, for apsidal positions, the instantaneous speed vector is orthogonal to the position vector.

Note that if $z(t)$ and $\dot{z}(t)$ are continuous and differentiable for every values of $t$, the differentiability domain of $r(t)$ is stricly limited to the real interval $] r_{m}, r_{M}[$.

To extract from Eq.(15) the radial celerity $\dot{r}(t)$ as a real-valued function, the square root has to be constructed with a pre-defined sign. To take account of the turn back in the radial motion, this sign changes when $r$ reaches the extremal values $r_{m}$ and $r_{M}$, which corresponds to change the square root determination.

We obtain:

$$
\dot{r}(t)= \pm \frac{L}{r(t)} \sqrt{f(r(t))}
$$

or:

$$
d r(t)= \pm \frac{L}{r} \sqrt{f(r)} d t
$$

The $+\operatorname{sign}$ is chosen when $r$ moves from $r_{m}$ to $r_{M}$ (increasing phase of the radial oscillation, $\left.d r(t)>0\right)$ and the - sign during the reverse motion (decreasing phase of the radial oscillation, $d r(t)<0$ ). To be more precise, we will index each phase of the motion by an integer number $k \in \mathbb{N}$, the even value $k=2 n$ corresponding to the decreasing phase of the $n$-th period (except for $n=0$ ) and the odd value $k=2 n+1$ corresponding to the increasing phase of the same period. With this convention, we then have:

$$
d r(t)=(-1)^{k+1} \frac{L}{r(t)} \sqrt{f(r(t))} d t
$$

and:

$$
\dot{r}(t)=(-1)^{k+1} \frac{L}{r(t)} \sqrt{f(r(t))}
$$

Eq.(21) gives implicitely the solution of the radial equation of motion Eq.(12).

\section{Orbital equation and r-parametrization of the motion}

In the $k$-phase of the motion, in every point except the apsidal ones, $\dot{r}(t)$ is a monotonic function (see Eq. (22)) and we can choose to parametrize the motion by $r$ rather than by $t$.

For instance:

$$
\frac{d \theta}{d r(t)}=\frac{\dot{\theta}}{\dot{r}}=(-1)^{k+1} \frac{1}{r \sqrt{f(r)}}
$$

where we have used Eq.(22) and Eq.(9).

Choosing a reference time $t_{0}$ during the same phase, we can write:

$$
\theta(r(t))=\theta\left(r_{0}\right)+(-1)^{k+1} \int_{r_{0}}^{r(t)} \frac{d \rho}{\rho \sqrt{f(\rho)}}
$$

with $r\left(t_{0}\right)=r_{0}$ and the orbital equation for this phase writes:

$$
\theta(r)=\theta\left(r_{0}\right)+(-1)^{k+1} \int_{r_{0}}^{r} \frac{d \rho}{\rho \sqrt{f(\rho)}}
$$

We have to be more careful if we want to use $r$ as a global parameter for the motion. Indeed, since $r(t)$ is a periodic function of $t, t(r)$ and every monotonic function of $t$, as $\theta$ (see Eq.(9)), will be multivalued. Therefore, at every $k$ phase of the motion will be attached a different branch of the function. 
For simplicity, we choose the initial condition:

$$
\left\{\begin{array}{c}
r(t=0)=r_{m} \\
\theta(t=0)=0
\end{array}\right.
$$

Then, the different determinations of $\theta(r)$ corresponding to each phase of the motion are given by:

In the first phase of the motion : $\theta(r(t))=g(r(t))$

In the second phase of the motion : $\theta(r(t))=\Phi-\int_{r_{M}}^{r(t)} \frac{d \rho}{\rho \sqrt{f(\rho)}}=2 \Phi-g(r(t))$

In the third phase of the motion : $\theta(r(t))=2 \Phi+g(r(t))$

where:

$$
g(r(t))=\int_{r_{m}}^{r(t)} \frac{d \rho}{\rho \sqrt{f(\rho)}}
$$

$\Phi=g\left(r_{M}\right)=\int_{r_{m}}^{r_{M}} \frac{d r^{\prime}}{r^{\prime} \sqrt{f\left(r^{\prime}\right)}}$ being the apsidal angle.

More generally, we will write:

$$
\text { In the } k \text {-th phase of the motion : } \theta_{k}(r(t))=2 n \Phi+(-1)^{k+1} g(r(t))
$$

where $n$ is the integral part of $\frac{k}{2}$.

From Eq.(22) and Eq. 23), we deduce the following expression for the instantaneous velocity in the $k$-th phase:

$$
\dot{z}(t)=\left((-1)^{k+1} \sqrt{f(r(t))}+i\right) \frac{L}{r^{2}(t)} z(t)=\frac{L}{r(t)}\left((-1)^{k+1} \sqrt{f(r(t))}+i\right) e^{i \theta_{k}(r(t))}
$$

$\theta_{k}(r)$ being given by Eq.(30).

Note that, since $\dot{z}=\lambda z$ where $\lambda$ has a nonzero imaginary part, Eq. (31) ensures the non-colinearity of $\vec{r}$ and $\dot{\vec{r}}$. In the sequel, we will have to use the vector $\dot{\vec{r}} \times \vec{L}$ which has the following complex correspondent :

$$
\dot{\vec{r}}(t) \times \vec{L} \rightarrow i L \dot{z}(t)=-\left(1+(-1)^{k} i \sqrt{f(r(t))}\right) \frac{L^{2}}{r^{2}(t)} z(t)
$$

We see immediately that $\vec{L} \times \dot{\vec{r}}$ and $\vec{r}$ are always linearly independent, except at the apsidal positions.

\section{PERES APPROACH TO THE FBRS VECTOR}

In the special case of the Kepler system $U(r)=-r^{-1}$, the system admits the Laplace-Runge-Lenz vector, $\overrightarrow{\mathcal{A}}_{L R L}$ $=\dot{\vec{r}} \times \vec{L}-\frac{1}{r} \vec{r}$, as a supplementary conserved quantity $\mathbb{\mathrm { B }}$. In the complex formulation used above, this gives :

$$
\overrightarrow{\mathcal{A}}_{L R L} \rightarrow \mathcal{A}_{L R L}=i L \dot{z}-\frac{1}{r} z
$$

Following Peres 19 , for the general central potential $U(r)$, we will then look for a supplementary conserved vector of the form:

$$
\overrightarrow{\mathcal{A}}=\dot{\vec{r}} \times \vec{L}\left(\frac{r^{2}}{L^{2}} a(r)\right)+b(r) \vec{r}
$$


whose associated complex correspondent is:

$$
\mathcal{A}=\left(\frac{r^{2}}{L^{2}} a(r)\right) i L \dot{z}+b(r) z
$$

$a(r)$ and $b(r)$ being two real valued functions that we will have to determine (the factor $\frac{r^{2}}{L^{2}}$ in the first term has been introduced for future convenience).

Using Eq.(32), we can rewrite $\mathcal{A}$ in the $k$-th phase, as:

$$
\mathcal{A}_{k}=\left(b(r)-a(r)+(-1)^{k+1} i \sqrt{f(r)} a(r)\right) z
$$

\section{Differential system for the coefficients of the FBRS vector}

On every time interval $I$ on which $\mathcal{A}$ is constant we must have:

$$
\dot{\mathcal{A}}=0
$$

that is, with Eq.(31):

$$
\begin{aligned}
0=\frac{d}{d t}( & \left.b(r)-a(r)+(-1)^{k+1} i \sqrt{f(r)} a(r)\right) z+\left(b(r)-a(r)+(-1)^{k+1} i \sqrt{f(r)} a(r)\right) \dot{z} \\
& \dot{r}\left(b^{\prime}(r)-a^{\prime}(r)+(-1)^{k+1} i\left(\sqrt{f(r)} a^{\prime}(r)+\frac{a(r) f^{\prime}(r)}{2 \sqrt{f(r)}}\right)\right) z \\
& +\left(b(r)-a(r)+(-1)^{k+1} i \sqrt{f(r)} a(r)\right)\left((-1)^{k+1} \sqrt{f(r)}+i\right) \frac{L}{r^{2}} z=0
\end{aligned}
$$

Using Eq.(22), this becomes:

$$
(-1)^{k+1} \sqrt{f(r)}\left(b^{\prime}(r)-a^{\prime}(r)+\frac{b(r)-2 a(r)}{r}\right)+i\left(\sqrt{f(r)} a^{\prime}(r)+\left(\frac{f^{\prime}(r)}{2 \sqrt{f(r)}}+\frac{f(r)-1}{r}\right) a(r)+b(r)\right)=0
$$

Consequently, in the $k$-phase of the motion, on the interval of radial values $] r_{m}, r_{M}$ [, we must have:

$$
\left\{\begin{array}{c}
a^{\prime}(r)-b^{\prime}(r)+\frac{2 a(r)-b(r)}{r}=0 \\
r f(r) a^{\prime}(r)+a(r)\left(r \frac{f^{\prime}(r)}{2}+f(r)-1\right)+b(r)=0
\end{array}\right.
$$

Extracting $a^{\prime}(r)$ from the second equation above and substituting in the first one, we obtain, in matrix form:

$$
\left(\begin{array}{l}
a(r) \\
b(r)
\end{array}\right)^{\prime}=\frac{-1}{r f(r)}\left(\begin{array}{cc}
r \frac{f^{\prime}(r)}{f^{2}}+f(r)-1 & 1 \\
r \frac{f^{\prime}(r)}{2}-f(r)-1 & f(r)+1
\end{array}\right)\left(\begin{array}{l}
a(r) \\
b(r)
\end{array}\right)
$$

\section{Exact solution}

Pere 19 , starting from a first order differential system equivalent to Eq.(41) transforms it in a second order differential equation for the coefficient $a(r)$. Nevertheless, the structure of this last equation is, at first sight, rather complicated and the author restricts its analysis to a characterization of the singularities.

Things are much more transparent if we choose to work with a slightly different unknown function. 
Indeed, it is readily seen that the first equation in Eq.(41) can be rewritten:

$$
r a^{\prime}(r)+2 a(r)=(r b(r))^{\prime}
$$

Introducing an auxiliary function $u(r)$ defined by:

$$
a(r)=u^{\prime}(r)
$$

the above equation Eq.(41) becomes:

$$
r u^{\prime \prime}(r)+2 u^{\prime}(r)=(r u(r))^{\prime \prime}=(r b(r))^{\prime}
$$

Then:

$$
b(r)=\frac{1}{r}(r u(r))^{\prime}+b_{0}=u^{\prime}(r)+\frac{1}{r} u(r)
$$

where the integration constant $b_{0}$ has been chosen equal to 0 .

We can report the expressions Eq.(44) and Eq.(46) of $a(r)$ and $b(r)$ in terms of $u(r)$ in the second equation of Eq.(41). This gives:

$$
\left(u^{\prime}(r)+\frac{1}{r} u(r)\right)^{\prime}=\frac{-1}{r f(r)}\left(u^{\prime}(r)\left(r \frac{f^{\prime}(r)}{2}-f(r)-1\right)+\left(u^{\prime}(r)+\frac{1}{r} u(r)\right)(f(r)+1)\right)
$$

or :

$$
u^{\prime \prime}(r)+\left(\frac{1}{r}+\frac{f^{\prime}(r)}{2 f(r)}\right) u^{\prime}(r)+\frac{1}{r^{2} f(r)} u(r)=0
$$

In a more compact form, we obtain the following second order differential equation for $u(r)$ :

$$
u^{\prime \prime}(r)-\left(\log \left(\frac{1}{r \sqrt{f(r)}}\right)\right)^{\prime} u^{\prime}(r)+\left(\frac{1}{r \sqrt{f(r)}}\right)^{2} u(r)=0
$$

or (see Eq.(29)):

$$
u^{\prime \prime}(r)-\frac{g^{\prime \prime}(r)}{g^{\prime}(r)} u^{\prime}(r)+\left(g^{\prime}(r)\right)^{2} u(r)=0
$$

The coefficients in Eq.(50) depending only on $g(r)$, if we define:

$$
u(r)=v(g(r))
$$

Eq.(50) becomes then:

$$
\left(g^{\prime}(r)\right)^{2} v^{(2)}(g(r))+g^{\prime \prime}(r) v^{(1)}(g(r))-\frac{g^{\prime \prime}(r)}{g^{\prime}(r)}\left(g^{\prime}(r) v^{(1)}(g(r))\right)+\left(g^{\prime}(r)\right)^{2} v(g(r))=0
$$

that is, simply:

$$
v^{(2)}(g(r))+v(g(r))=0
$$


where $v^{(n)}(x)=\frac{d^{n} v}{d x^{n}}$.

The resolution of Eq.(53) is immediate and gives:

$$
u(r)=\alpha \cos g(r)+\beta \sin g(r), \quad \alpha, \beta \in \mathbb{R}
$$

Reporting this result in Eq.(44) and Eq.(46), we finally have:

$$
\left\{\begin{array}{c}
a(r)=\frac{1}{r \sqrt{f(r)}}(\beta \cos g(r)-\alpha \sin g(r)) \\
b(r)=a(r)+\frac{1}{r}(\alpha \cos g(r)+\beta \sin g(r))
\end{array}, \quad \alpha, \beta \in \mathbb{R}\right.
$$

that is:

$$
\left\{\begin{array}{c}
a(r)=\frac{\gamma}{r \sqrt{f(r)}} \cos (g(r)+\phi) \\
b(r)=a(r)+\frac{\gamma}{r} \sin (g(r)+\phi)
\end{array}, \quad \gamma \in \mathbb{R}, \phi \in[0,2 \pi[\right.
$$

\section{Discontinuous behavior of $\mathcal{A}$}

The preceding result Eq. 56 ) determines completely the form of $\mathcal{A}$ on $] r_{m}, r_{M}[$ during a given phase of the motion. Indeed, inserting Eq.(56) in Eq.(36), we obtain:

$$
\mathcal{A}_{k}=\frac{\gamma}{r}\left(\sin (g(r)+\phi)+(-1)^{k+1} i \cos (g(r)+\phi)\right) z(r)=(-1)^{k+1} i \gamma e^{(-1)^{k} i(g(r)+\phi)} e^{i \theta_{k}(r)}
$$

Using Eq.(30), this becomes:

$$
\mathcal{A}_{k}=(-1)^{k+1} i \gamma e^{(-1)^{k} i(g(r)+\phi)} e^{i\left(2 n \Phi+(-1)^{k+1} g(r)\right)}=(-1)^{k+1} \gamma i e^{(-1)^{k} i \phi+2 n i \Phi}
$$

where $n$ is the integer part of $\frac{k}{2}$.

Under a more detailed form, we have:

$$
\left\{\begin{array}{l}
\mathcal{A}_{2 n}=-\gamma e^{i \frac{\pi}{2}+i \phi+2 n i \Phi} \\
\mathcal{A}_{2 n+1}=\gamma e^{i \frac{\pi}{2}-i \phi+2 n i \Phi}
\end{array}\right.
$$

The integration constants $\gamma$ and $\phi$ for and determine then respectively the modulus and argument of the $\overrightarrow{\mathcal{A}}$ vector affix. The freedom in the choice of the parameters $\gamma$ and $\phi$ induces that $\overrightarrow{\mathcal{A}}$ can be identified with any vector of the plane. We have the same type of result for the specific Kepler problem. Indeed, in this case, we can add to the Laplace-Runge-Lenz vector $\overrightarrow{\mathcal{A}}_{K}$ a second conserved vector, $\overrightarrow{\mathcal{S}}_{K}=\vec{L} \times \overrightarrow{\mathcal{A}}_{K}$, the so-called Hamilton vector 1 阳 1 , whose constancy is a direct consequence of these ones of $\vec{L}$ and $\overrightarrow{\mathcal{A}}_{K}$. Any linear combination of this two orthogonal vectors being conserved, we can build a conserved vector corresponding to any vector of the plane. $\overrightarrow{\mathcal{S}}_{K}$ and $\overrightarrow{\mathcal{A}}_{K}$ are particularly interesting choices because they give the directions of the minor and major axes of the elliptical trajectory.

If we want that the functional forms of $a(r)$ and $b(r)$ being globally defined during all the motion, we have to keep the same values for $\gamma$ and $\phi$ in every phase. With the choice $\phi=\frac{\pi}{2}$, Eq. (59) becomes:

$$
\mathcal{A}_{2 n}=\mathcal{A}_{2 n+1}=\gamma e^{2 n i \Phi}
$$

$\overrightarrow{\mathcal{A}}$ is then constant when we pass from a even phase to the following odd one, that is when we cross the apocenter. But after a complete period of oscillation, when we reach the pericenter again, we pass from $k=2 n$ to $k=2 n+1$. At this moment the value of $\mathcal{A}_{2 n+1}$ change to $\mathcal{A}_{2 n+1}$ with:

$$
\mathcal{A}_{2 n+2}=\mathcal{A}_{2 n+1} e^{2 i \Phi}
$$

This corresponds to a $2 \Phi$ rotation of the assgciated vector. We recover here the discontinuity jumps (observed by Serebrennikov, Shabad, Buch and Denman $17-18$ and Peres 19 and first studied in a detailed by Holas and March 21 ) which make FBRS vector only a piecewise conserved quantity: the perihelion vector $\mathcal{A}$ presents discontinuities at each pericenter (with the choice made here and at each apocenter if we choose the initial condition $r(t=0)=r_{M}$ ) corresponding to a rotation of two times the apsidal angle. 
For $\Phi=\pi$, which is the case of the Kepler problem $U(r)=-r^{-1}$ for every values of the characteristic parameters of the motion $L$ and $E, \overrightarrow{\mathcal{A}}$ is a true vector conserved quantity and is identical to the usual Laplace-Runge-Lenz vector $\overrightarrow{\mathcal{A}}_{K}$. Indeed, in this case, with $E<0$, introducing Clairaut's variablet $u=\frac{1}{r}$, we have (see Eq. 29.) and(see Eq.(16)):

$$
g(r)=-\frac{L}{\sqrt{2}} \int_{u_{m}=\frac{1}{r_{m}}}^{u} \frac{d v}{\sqrt{-|E|+v-\frac{L^{2}}{2} v^{2}}}
$$

which gives 29 for $1-2 L^{2}|E|=e^{2}>08$ :

$$
g(r)=\left[\arg \sin \left(\frac{1-L^{2} v}{e}\right)\right]_{u_{m}}^{u=\frac{1}{r}}
$$

Since $r_{m}$ is the smallest root of $f(r)=\frac{2 r^{2}}{L^{2}}\left(-|E|+\frac{1}{r}-\frac{L^{2}}{2 r^{2}}\right)=0$, that is, $u_{m}=\frac{1}{r_{m}}$ is the greatest root of $-|E|+u-\frac{L^{2}}{2} u^{2}=0$, we obtain:

$$
u_{m}=\frac{1+e}{L^{2}}
$$

and:

$$
g(r)=\arg \sin \left(\frac{1-\frac{L^{2}}{r}}{e}\right)+\frac{\pi}{2}
$$

Then (see Eq.(56) with $\left.\phi=\frac{\pi}{2}\right)$ :

$$
\left\{\begin{array}{c}
a(r)=\frac{\gamma}{r \sqrt{f(r)}} \cos \left(g(r)+\frac{\pi}{2}\right)=-\frac{\gamma}{r \sqrt{f(r)}} \sqrt{1-\left(\frac{r-L^{2}}{e r}\right)^{2}}=-\gamma \frac{L^{2}}{e r^{2}} \\
b(r)=a(r)+\frac{\gamma}{r} \sin \left(g(r)+\frac{\pi}{2}\right)=-\gamma \frac{L^{2}}{e r^{2}}-\frac{\gamma}{r} \frac{r-L^{2}}{e r}=-\gamma \frac{1}{e r}
\end{array}\right.
$$

Taking $\gamma=-e$, we recover the coefficients of the usual Laplace-Runge-Lenz vector (see Eq.(33):

$$
\left\{\begin{array}{c}
a(r)=\frac{L^{2}}{r^{2}} \\
b(r)=\frac{1}{r}
\end{array}\right.
$$

In the isotropic harmonic oscillator problem (Hooke's problem) $\Phi=\frac{\pi}{2}$, for every $L$ and $E$. The global direction is then conserved but the sense of $\overrightarrow{\mathcal{A}}$ is alterned at each pericenter crossing : $\overrightarrow{\mathcal{A}} \rightarrow-\overrightarrow{\mathcal{A}} 17 \mathbf{1 8}$. The generalized Hamilton vector $\overrightarrow{\mathcal{S}}=\vec{L} \times \overrightarrow{\mathcal{A}}$ is subject to the same phenomenon : $\overrightarrow{\mathcal{S}} \rightarrow-\overrightarrow{\mathcal{S}}$. As for the Fradkin tensor $\mathcal{T}=\frac{1}{2} \dot{\vec{r}} \otimes \dot{\vec{r}}+\frac{\omega^{2}}{2} \vec{r} \otimes \vec{r}$ , recalling that it can be written as $\mathbb{1} \mathcal{T}=\frac{\omega^{2}}{2} \overrightarrow{\mathcal{A}} \otimes \overrightarrow{\mathcal{A}}+\frac{1}{2|\mathcal{A}|^{2}} \overrightarrow{\mathcal{S}} \otimes \overrightarrow{\mathcal{S}}$, we see immediately that it's a global invariant of the motion.

As established by Bertrand25.26 more than one century ago, Kepler and Hooke potentials are the only central potentials for which the apsidal angle is commensurable with $\pi$ for every values of the initial parameters of the motion. For all the other central potentials, this condition, which is necessary for theclosure of the orbit, is obtained only for specific values of $E$ and $L$. In these cases, as established by Holas and March21, it is still possible for an orbit of multiplicity $n$ to build global geometrical invariants in form of $n$-arm stars by using the $n$ distinct FBRS vectors associated to the system. Nevertheless, as we have seen before, the existence of a general (that is for every initial condition) true invariant vector or tensor is a specificity of Kepler and Hooke problems respectively.

${ }^{1}$ P. G. L. Leach and G. P. Flessas, " Generalisations of the Laplace-Runge-Lenz vector ," J. Nonlin. Math. Phys. 10, 340-423 (2003). 
${ }^{2}$ H. Goldstein, "Prehistory of the "Runge-Lenz" vector," Am. J. Phys. 43, 737-738 (1975).

${ }^{3}$ H. Goldstein, "More on the prehistory of the Laplace or Runge-Lenz vector ," Am. J. Phys. 44, 1078-1082 (1976).

${ }^{4}$ E.T. Whittaker, A treatise on the analytical dynamics of particles \&5 rigid bodies (Cambridge University Press, 1999).

5 M. Bander and C. Itzykson, "Group theory and the hydrogen atom (I) an (II)," Rev. Mod. Phys. 38, 330-358 (1966).

${ }^{6}$ D. F. Greenberg, "Accidental degeneracy, " Am. J. Phys. 34, 1101-1109 (1966).

${ }^{7}$ H.V. McIntosh, in Group theory and its applications (vol II), ed. E. M. Loeb (Academic Press, 1971).

8 B. Cordani, The Kepler problem (Birkhäuser, 2003).

9 J. M. Jauch and E. L. Hill, "On the problem of degeneracy in quantum mechanics," Phys. Rev. 57, 641-645 (1940).

10 D. M. Fradkin, "Three-dimensional isotropic harmonic oscillator and SU," Am. J. Phys. 33, 207-211 (1965).

11 J. Sivardière, "Comments on the dynamical invariants of the Kepler and harmonic motions," Eur. J. Phys. 13, 64-69 (1992).

12 H. Bacry, H. Ruegg and J.M. Souriau, "Dynamical groups and spherical potentials in Classical Mechanics", Comm. Math. Phys. 3, 323-333 (1966).

13 D. M. Fradkin, "Existence of the dynamics symmetries $\mathrm{O}_{4}$ and $\mathrm{SU}_{3}$ for all classical central potential problems," Prog. Theor. Phys. 37, 798-812 (1967).

14 N. Mukunda, "Dynamical symmetries and classical mechanics," Phys. Rev. 155, 1383-1386 (1967).

15 J.-M. Souriau, Structure des systèmes dynamiques, (Dunod, 1969).

16 P. Stehle and M. Y. Han, "Symmetry and degeneracy classical mechanics," Phys. Rev. 159, 1076-1082 (1967).

17 V. B. Serebrennikov and A. E. Shabad, "Method of calculation of the spectrum of a centrally symmetric Hamiltonian on the basis of approximate $\mathrm{O}_{4}$ and $\mathrm{SU}_{3}$ symmetries ," Theor. Math. Phys. 8, 644 (1971).

18 L. H. Buch and H.H. Denman, "Conserved and piecewise-conserved Runge vectors for the isotropic harmonic oscillator," Am. J. Phys. 43, 1046-1048 (1975).

19 A. Peres, "A classical constant of motion with discontinuities," J. Phys. A 12, 1711-1713 (1979).

20 T. Yoshida, "Two methods of generalisation of the Laplace-Runge-Lenz vector," Eur. J. Phys.. 8, 258-259 (1987).

21 A. Holas and N.H. March, "A generalisation of the Runge-Lenz constant of classical motion in central potential," J. Phys. A 23, 735-749 (1990).

22 C.C. Yan, "Determination of vector constant of motion for a particle moving in a conservative central force field," J. Phys. A 24, 4731-4738 (1991).

23 A. Holas and N.H. March, "How many vector constants of motion exist for a particle moving in a central potential ?" J. Phys. A 27, 2615-2617 (1994).

24 A. Ballesteros, A. Enciso, F. J. Herranz and O. Ragnisco, "Hamiltonian systems admitting a Runge-Lenz vector and a optimal extension of Bertrand's theorem to curved manifolds," preprint arXiv 0810.0999

25 J. Bertrand "Théorème relatif au mouvement d'un point attiré vers un centre fixe", C. R. Acad. Sci. 77, 849-853 (1873).

${ }^{26}$ Y. Grandati, A. Bérard and F. Menas, "An alternative proof of Bertrand's theorem," Am. J. Phys. 76, 782-787 (2008).

27 Y. Grandati, A. Bérard and H. Mohrbach, "Bohlin-Arnold-Vassiliev's duality and conserved quantities," preprint arXiv 0803.2610

28 Y. Grandati, A. Bérard and H. Mohrbach, "Duality properties of generalized Gorringe-Leach equations," preprint arXiv 0712.3338, submitted to Celest. Mech.

29 I. S. Gradshteyn, I. M. Ryzhik and A. Jeffrey, Table of integrals, series and products (Academic Press, 2000). 